\title{
Is Health-Related Quality of Life after Radical Cystectomy Using Validated Questionnaires Really Better in Patients with Ileal Orthotopic Neobladder Compared to lleal Conduit: A Meta-Analysis of Retrospective Comparative Studies
}

\author{
Maria A. Cerruto ${ }^{a}$ Carolina D'Elia $^{a}$ Salvatore Siracusano ${ }^{a}$ Antonio B. Porcaro ${ }^{a}$ \\ Giovanni Cacciamani $^{\mathrm{a}} \quad$ Davide De Marchi $^{\mathrm{a}} \quad$ Mauro Niero $^{\mathrm{b}} \quad$ Cristina Lonardi $^{\mathrm{b}}$ \\ Massimo lafrate ${ }^{c}$ Pierfrancesco Bassi ${ }^{d} \quad$ Emanuele Belgrano $^{e} \quad$ Ciro Imbimbo $^{f}$ \\ Marco Racioppi $^{\text {d }}$ Renato Talamini ${ }^{9}$ Stefano Ciciliato ${ }^{\mathrm{e}}$ Laura Toffoli $^{\mathrm{e}}$ \\ Michele Rizzo ${ }^{e} \quad$ Francesco Visalli $^{\mathrm{e}} \quad$ Paolo Verze $^{\mathrm{f}} \quad$ Walter Artibani $^{\mathrm{a}}$
}

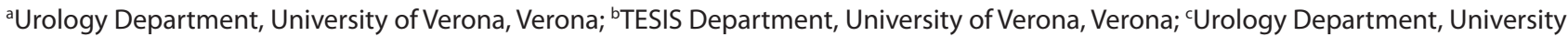
of Padua, Padua; dUrology Department, Catholic University Policlinico Gemelli, Rome; eUrology Department, Trieste University, Trieste; fUrology Department, University of Naples, Naples; 9Unit of Epidemiology and Biostatistics, IRCCS-CRO, Aviano, Italy

\section{Key Words}

Radical cystectomy $\cdot$ Health related quality of life $\cdot$

Urinary diversion $•$ Ileal conduit $\bullet$ Neobladder

\begin{abstract}
Introduction: From the most recent systematic revision of the literature, an orthotopic neobladder would seem to show marginally better health related quality of life (HRQoL) scores compared with an ileal conduit. The aim of this study was to review all relevant published studies about the comparison between ileal orthotopic neobladder (IONB) and ileal conduit using validated HR-QoL questionnaires. Materials and Methods: Studies were identified by searching multiple literature databases. Data were synthesized using meta-analytic methods conformed to the PRISMA statement. Results: The literature search identified 10 papers; pooled effect sizes of combined quality of life outcomes for ileal conduit versus IONB showed a significantly better HR-
\end{abstract}

\section{KARGER}

(C) 2016 S. Karger AG, Basel

Fax +4161306 1234

E-Mail karger@karger.com

www.karger.com
Accessible online at:

www.karger.com/cu
QoL in patients with IONB (Hedges' $g=0.278 ; p=0.000) ;$. The present study has an important limitation due to the type of the analyzed comparative studies, all retrospective and not randomized. Conclusion: This meta-analysis of not-randomized, retrospective comparative studies on the impact of ileal conduit versus IONB on HR-QoL showed a significant advantage of IONB subgroups.

Copyright $\odot 2016$ S. Karger AG, Basel

\section{Introduction}

Radical cystectomy remains the curative treatment of choice for muscle-invasive bladder cancer [1]; moreover radical cystectomy [2] should be considered in T1G3 bladder cancer associated with concurrent bladder carcinoma in situ, multiple and/or large T1G3 and/or recurrent T1G3, T1G3 with carcinoma in situ in prostatic urethra and it is recommended in BCG refractory tumors. 
After radical cystectomy, virtually every segment of gastro-intestinal tract has been used to create a urinary diversion (UD). However, historically an ileal conduit (IC) has been the mainstay of UD, but over the past two decades an orthotopic neobladder (ONB) has become the "gold standard" for urinary tract reconstruction after radical cystectomy [3]. Several factors may affect the choice of UD, including patient factors (choice, anatomy, motivation, comorbidities, manual dexterity, body habitus, general health, performance status, mental capacity), physician factors (expertise, available medical/nursing support), and general factors [health related quality of life (HR-QoL), available caregivers, cancer extent, life expectancy, lifestyle, cultural factors] [4]. Actually, the impact of different types of UD on patients' HR-QoL represents one of the most important issues in the choice of the best reservoirs or conduits. There are few and contrasting data comparing HR-QoL, using validated questionnaires, in different types of ONB each other. Arata et al. [5] compared HR-QoL in 78 patients with either ileal ONB (IONB) or sigmoidal ONB (SONB) using the EORTC QLQ-C30. They found that the QoL score concerning voiding conditions, daytime, and night time continence and quantity of pad showed a better score in the ileal group. Concerning the satisfaction, patients in the sigmoidal group expressed less satisfaction than their preoperative expectations; moreover, concerning several postoperative conditions, IONB seemed superior to SONB. Miyake et al [6], using the Short Form-36, showed no significant differences in all scores between male SONB and IONB, whereas 3 scores (role-physical functioning, role-emotional functioning, and social functioning) in female patients with SONB were significantly superior to those with IONB. However, multivariate analysis revealed that, in despite of the lack of significant impact of the type of ONB on HR-QoL, elderly patients and/or patients who could not void spontaneously failed to achieve a favourable HR-QoL postoperatively. During a long-term follow-up, the voiding status in patients with IONB appeared to tend to be impaired compared to SONB, remaining comparable in satisfactory aspects [7]. From the most recent systematic revision of the literature, an ONB (without differentiation in type) would seem to show marginally better HR-QoL scores compared to an IC, especially when considering younger and fitter patients [8]. There are no cumulative data on the comparison of HR-QoL in patients with IC versus a specific ONB. The aim of this study was to review of all relevant published studies on the comparison between IONB and IC using validated HR-QoL questionnaires.

\section{Materials and Methods}

\section{Literature Search Strategy}

Studies were identified by searching multiple literature databases, including MEDLINE, CINAHL (Cumulative Index to Nursing and Allied Health Literature), the Cochrane Library, PubMed. References from retrieved articles were searched manually. The "related articles" function in PubMed was also used. These databases were analyzed from the earliest report of QoL in patients with UD from 1980 to February 2015.

The following keywords were used in all searches: "quality of life", "urinary diversion", "ileal conduit", "neobladder", "ileal neobladder", "orthotopic neobladder", "ileal orthotopic neobladder". In the absence of randomized clinical trials, queries were limited to both retrospective and prospective comparative full text peer-review papers published in the English language, involving human subjects who underwent IC versus IONB.

The literature search was conducted independently by 2 investigators of the research team. Data extraction was recorded on customized tables; disagreements were solved though consensus. The data items extracted were: first author, country, journal, year of publication, study design (retrospective versus prospective), setting (single center versus multi-center) number of patients, percentage of female patients, age (years), follow-up (months), percentage of patients in $\leq$ pT2 bladder cancer, type of validated HR-QoL questionnaire used. Within each domain, data were further categorized into specific outcomes according to the different HR-QoL instrument used.

\section{Statistical Analyses}

Data were synthesized using meta-analytic methods [9]. The standard mean difference, or the effect size between the ONB and the IC urinary diversion, was calculated using Hedges' $g$ unbiased approach. Calculation of the effect sizes was based on means, differences in mean scores, $\mathrm{p}$ value, and simple sizes of the groups. Data were statistically pooled by the standard meta-analysis approach, meaning that studies were weighted by the inverse of the sampling variance. It was applied a test of heterogeneity was applied and the $\mathrm{I}^{2}$ statistic computed. The $\mathrm{I}^{2}$ statistic indicates the proportion of total variation among the effect estimates attributed to heterogeneity rather than sampling error, and has the advantage to being intrinsically independent of the number of the studies. When the test of heterogeneity was not significant $(p>0.05)$ and $\mathrm{I}^{2}$ was less than 30 per cent $[5,6]$, a fixed-effects model was adopted for evaluation of the results; otherwise, it was used a random-effects model. Several characteristics within the 2 analyzed patients' groups (IONB and IC) were identified and their effects on outcomes were examined. Categorical characteristics were treated as moderators and effectiveness was compared across subgroups formed by these moderators. Continuous characteristics were examined as covariates using random effects (method of moments) meta-regression. We also assessed publication bias using the Egger's $t$ test and funnel plots with significance values based on 1-tailed $p$ values $[10,11]$. Comprehensive Meta-Analysis V. $2^{\odot}$ software (BIOSTAT, Inc. Comprehensive Meta-Analysis V.2 Software Version V.2.Englewod, NJ: BIOSTAT, Inc; 2009) was used for statistical analyses. Statistics reported in this meta-analysis are conformed to the Preferred Reporting Items for Systematic Reviews and Meta-Analyses (PRISMA) statement [12]. Based on conventional standards, effect sizes of $\mathrm{g}$ equal to $0.20,0.50$, and 0.80 were considered small, medium, and large, respectively [13]. 


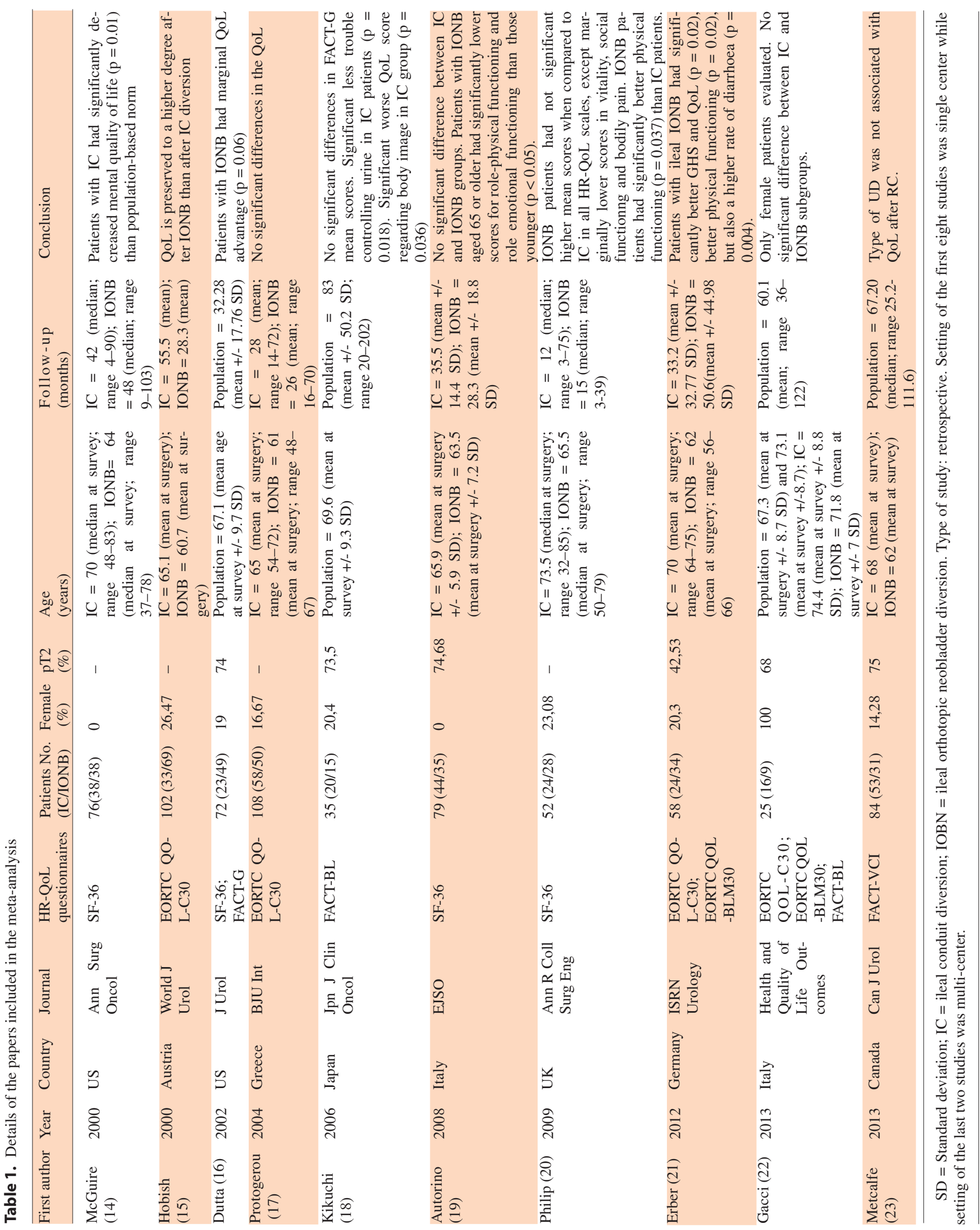




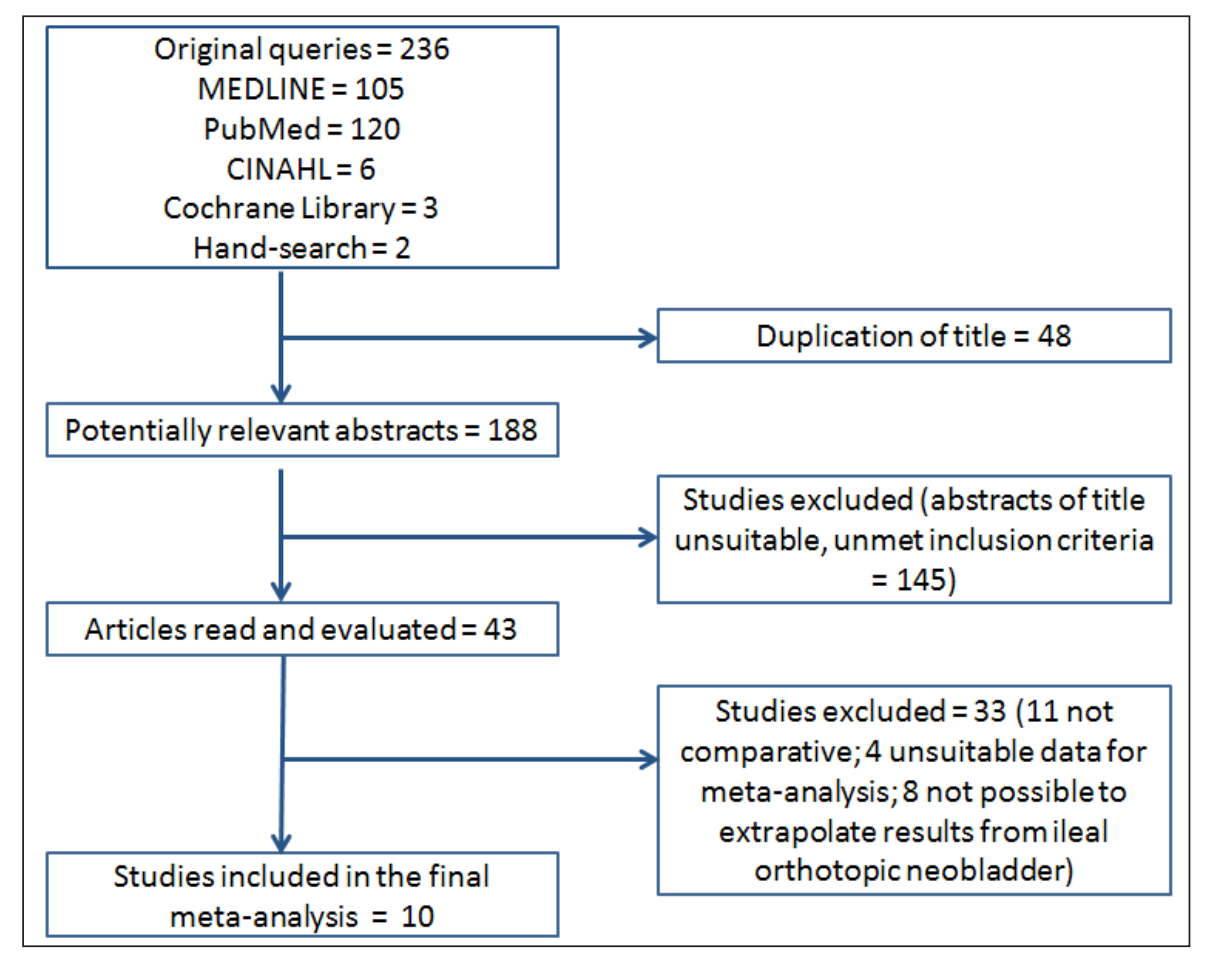

Fig. 1. Articles identified and evaluated during the review process for meta-analysis.

\section{Results}

Initial queries identified a total of 236 articles from all databases and search methods. Comparison of the retrieved titles identified 48 studies that were duplicates, thus leaving 188 abstracts for further evaluation (fig. 1).

The literature search identified 10 papers [14-23] that reported a HR-QoL comparison between IC and IONB using at least a validate questionnaire, suitable for the meta-analysis. Table 1 shows all details from the studies included in the meta-analysis.

All papers were retrospective, coming from different countries: 2 from Italy [19, 22], 2 from United States [14, 16], 1 from Austria [15], 1 from Canada [23], 1 from Germany [21], 1 from Greece [17], 1 from Japan [18], 1 from United Kingdom [20]. All except two [22, 23] had single center setting. Table 2 shows all different validated HRQoL tools used in the retrieved articles for meta-analysis. The Short Form-36 is a generic HR-QoL instrument that contains 36 questions assessing 8 aspects of HR-QoL, including physical functioning, role-physical functioning, role-emotional functioning, vitality, mental health, social functioning, bodily pain and general health [24]. These scales can also be grouped into physical and mental components scores. The higher the score is, the bet- ter the results are [25]. The European Organization for the Research and Treatment of Cancer Quality of Life Core Questionnaire (EORTC QLQ-C30) is a 30-item questionnaire composed of multi-item scales and single items reflecting the multidimensionality of the QoL construct $[35,36]$. It comprises 5 functional scales covering physical, role, emotional, cognitive and social aspects, and one scale of global health status/QoL (GQoL). It also includes 3 multi-item symptom scales of fatigue, nausea/ vomiting and pain, and six single items that deal with dyspnea, insomnia, appetite loss, constipation, diarrhea and financial difficulties caused by the disease or its treatment. For functional and overall scales higher scores represent a better outcome on QoL, whereas for symptom and single-item scales higher scores correspond to more problems and a reduced QoL. The EORTC QLQBLM30 is a module from the EORTC that specifically evaluates the impact of radical cystectomy and reconstructive surgery in terms of HR-QoL. For the symptoms/single items, a higher score means a higher level of symptoms/problems. The Functional Assessment of Cancer Therapy-Bladder (FACT-BL) is an assessment tool evaluating patients' bladder cancer specific HR-QoL [26]. It includes physical, social/family, emotional, and functional well-being (these domains together constitute 


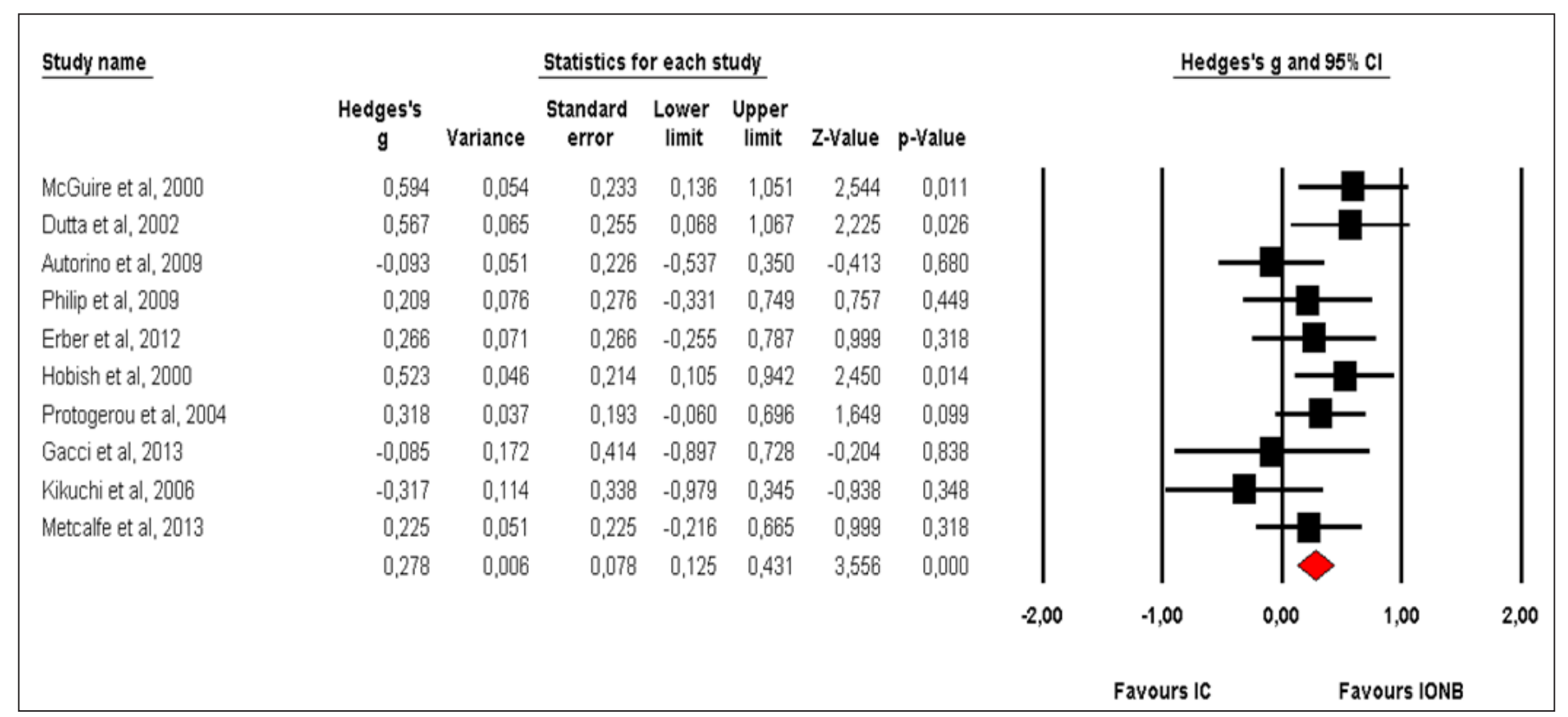

Fig. 2. Forest plot of overall combined HR-QoL domains for IC vs. IONB. A fixed-effects model was used for meta-analysis because $\mathrm{I}^{2}$ $=19.382 \%$. Standard mean difference estimates for individual papers, calculated using Hedges' g, are shown in boxes and the summary of effect as a diamond; $95 \%$ CI indicates $95 \%$ confidence interval.

Table 2. Different validate HR-QoL instruments used in the retrieved articles for meta-analysis

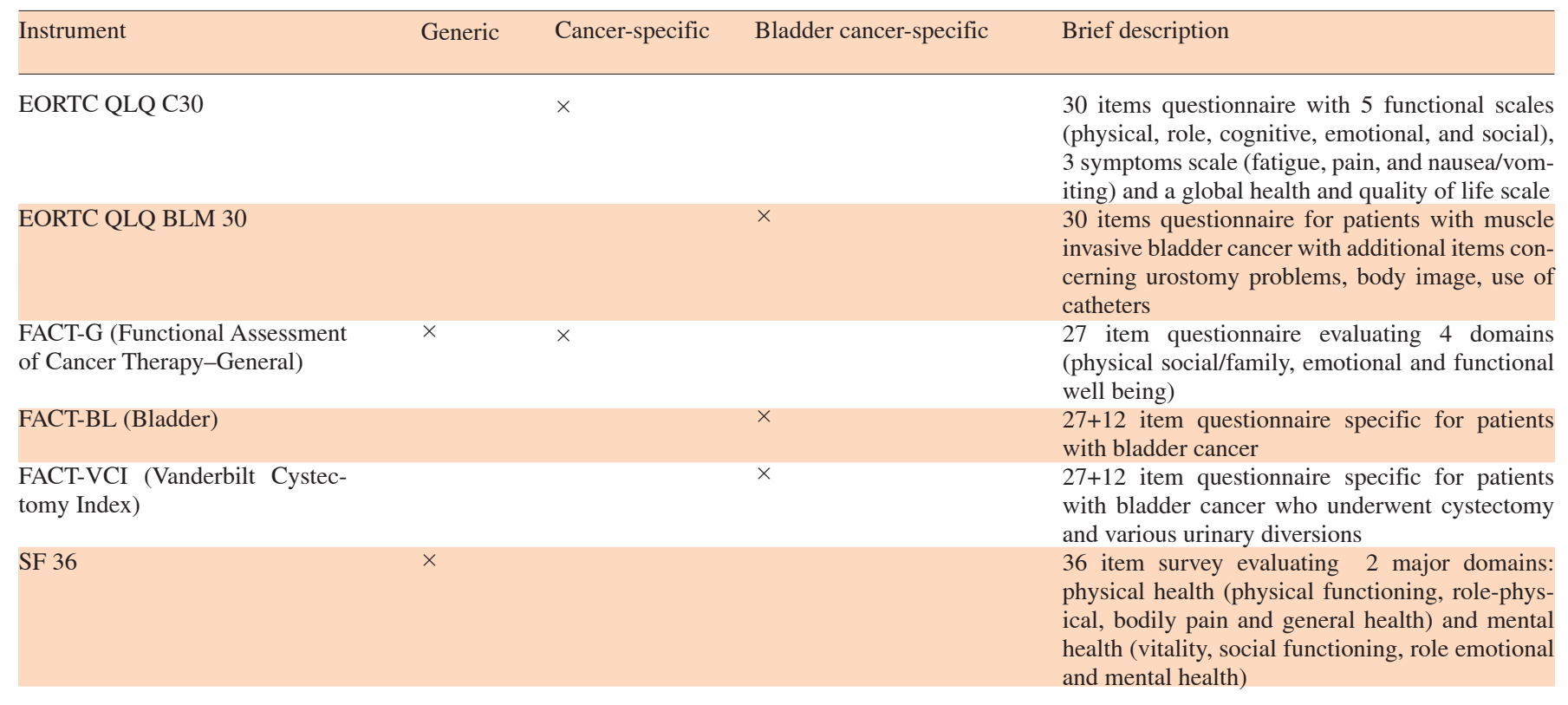

the FACT-G) that are relevant to all cancer patients. It also includes a subscale labelled "additional concerns" that is comprised of items relevant to patients treated with radical cystectomy and urinary diversion. The survey is composed of 39 questions. A total of 17 additional questions were added to the FACT-G to create the Van- 


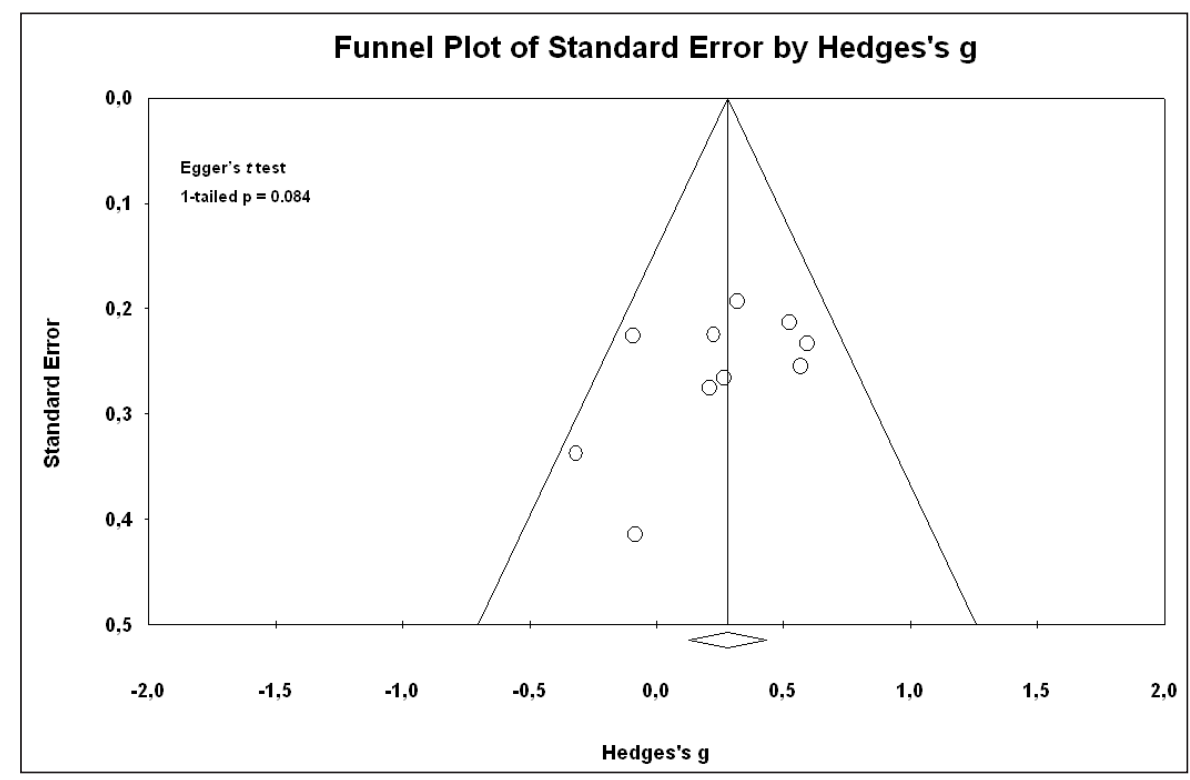

Fig. 3. Funnel plot with Egger's $t$ test for publication bias of overall combined selected papers for meta-analysis.

derbilt Cystectomy Index (FACT-VCI) [26]. Emotional and physical well-being lower scores match to higher HR-QoL; high social/family and functional scores indicate a high level of HR-QoL. According to the different validated HR-QoL tool used, 4 employed the EORTC QOL-C30 [15, 17, 21, 22], 4 the FACT (either G [16, 22], bladder [18, 22], or Vanderbilt Cystectomy Index [23]), 4 the social aspects $[14,16,19,20]$, and 2 the EORTC QoL-BLM30 [21, 22].

Overall 691 patients were analysed (333 IC and 358 IONB); $59 \%$ of patients were males with a population having a range age of 37 years up to 79 years and a range follow-up of 9 up to 202 months.

Pooled effect sizes of combined HR-QoL outcomes for IC versus IONB showed significantly better QoL in the IONB group [Hedges' $g=0.278(0.125 / 0.43195 \% \mathrm{CI})$; $\mathrm{p}=0.000$ ] (fig. 2). Figure 3 showed the Funnel plot with Egger's t test for publication bias. Table 3 shows the categorical moderators analyses (table 3 ). The overall pooled effect sizes analysis of the 8 aspects of the SF-36 showed a better HR-QoL in IONB group [Hedges' $\mathrm{g}=0.316$ $(0.176 / 0.45795 \% \mathrm{CI}) ; \mathrm{p}=0.000$ ] (fig. 4), mainly concerning the physical functioning $(\mathrm{p}=0.000)$ and the general health (0.007) domains. Analysing only the physical and the mental components scores of the Short Form-36, the significant favourable impact of IONB on patients' HR-QoL is confirmed [Hedges' $g=0.320(0.022 / 0.619$
$95 \% \mathrm{CI}$ ) $; \mathrm{p}=0.035]$ (fig. 5). In IONB group all functional scales from the EORTC QLQ-C30 [Hedges' $\mathrm{g}=$ $0.458(0.358 / 0.59995 \% \mathrm{CI}) ; \mathrm{p}=0.000$ ] (fig. 6) were significantly better, as well as all functional scales [Hedges' $\mathrm{g}=0.317$ (0.236/0.398 95\% CI); $\mathrm{p}=0.000$ ] (fig. 7) but one (diarrhea, $\mathrm{p}=0.981$ ). Any significant difference in the BI aspect scores from EORTC QLQ-BLM30 were observed in IC patients' compared with IONB group (fig. 8). There were no significant differences in all FACT-G aspects scores between IC and IONB groups (fig. 9).

\section{Discussion}

The current meta-analysis examined 10 comparative retrospective cross-sectional studies [14-23] addressing the impact of IC on patients' HR-QoL compared with IONB. From the pooled effect sizes analyses the overall HR-QoL appears to be significantly more favourable in the IONB group. Possible reason of this result may be found from the moderators analyses. Actually age, gender, type of validate questionnaire used and follow-up length may represent important factors affecting the impact of the type of UD on patients' HR-QoL.

Concerning the age, in our analysis, younger patients population $(<65$ years $)$ in IONB group significantly affected HR-QoL in favour of the orthotopic diversion 


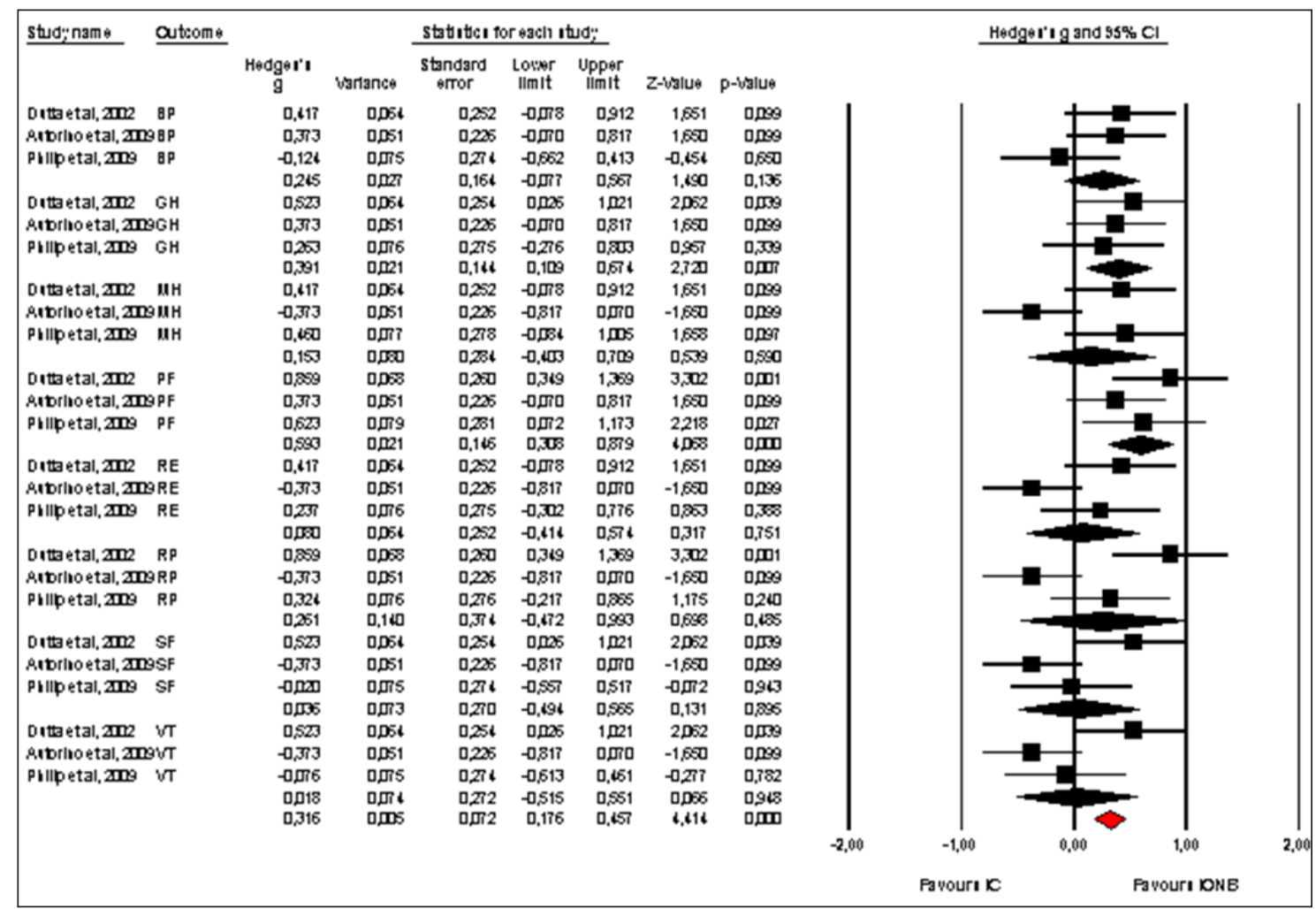

Fig. 4. Forest plot of pooled effect sizes of the 8 aspects of the SF-36. A random-effects model was used for meta-analysis because $\mathrm{I}^{2}=61.839 \%$. Standard mean difference estimates for individual papers, calculated using Hedges' g, are shown in boxes and the summary of effect as a diamond; 95\% CI indicates 95\% confidence interval.

compared to IC. Actually in all the analyzed studies, IC patients showed a mean/median age more than 65 years, older than IONB patients in 6 case series [14, 15, 17, 19, 21, 23]. Saika et al [27] examining HR-QoL in 78 patients (56 IC vs. 22 ONB) using the EORTC QLQ-C30, observed that in elderly patients (aged 75 years or older) no significant difference appeared among UD subgroups in any QoL area. Sogni et al. [28], studying 34 elderly patients (aged 75 years or older), 16 with IC and 18 with ONB, showed higher, GQoL scores in ONB group compared to IC, but this difference was not statistically significant $(\mathrm{p}=0.16)$. All the other scores were comparable [28]. Actually in patients with any type of ONB, HR-QoL, mainly those associated with physical and social functions, appeared to be significantly impaired in elderly patients and/or in those not able to spontaneously void [6]. Autorino et al. [19] observed that patients with IONB aged 65 or older had significantly lower scores for role-physical functioning and role emotional functioning than those younger $(p<0.05)$.

Health Related Quality of Life of Ileal Conduit vs. Ileal Orthotopic Neobladder
Regarding the impact of the gender on HR-QoL after radical cystectomy, in our analysis, all studies but one [22] showed a study populations with more than $65 \%$ men, with an overall better HR-QoL in favour of the ONB subgroup (Hedges' $\mathrm{g}=0.291, \mathrm{p}=0.000$ ). Performing a meta-regression of our data a light, non significant, slope in favour of male gender emerged. Actually, there are sparse information in the literature. Gacci et al. [22] in a series of only long-term disease-free female survivors after radical cystectomy, did not find significant difference between IC and IONB subgroups; in contrast, compared with those who underwent IC or IONB, women with cutaneous ureterostomy endorsed a worse HR-QoL, mostly due to the worse physical and emotional perception of their body image. In a more recent study, Rouanne et al. [29], examined 31 women with IONB after radical cystectomy for bladder cancer and observed that, at a mean follow-up of 5.7 years, IONB reconstruction provided long term satisfaction with maintained health related quality of life.

Curr Urol 2016;10:57-68 


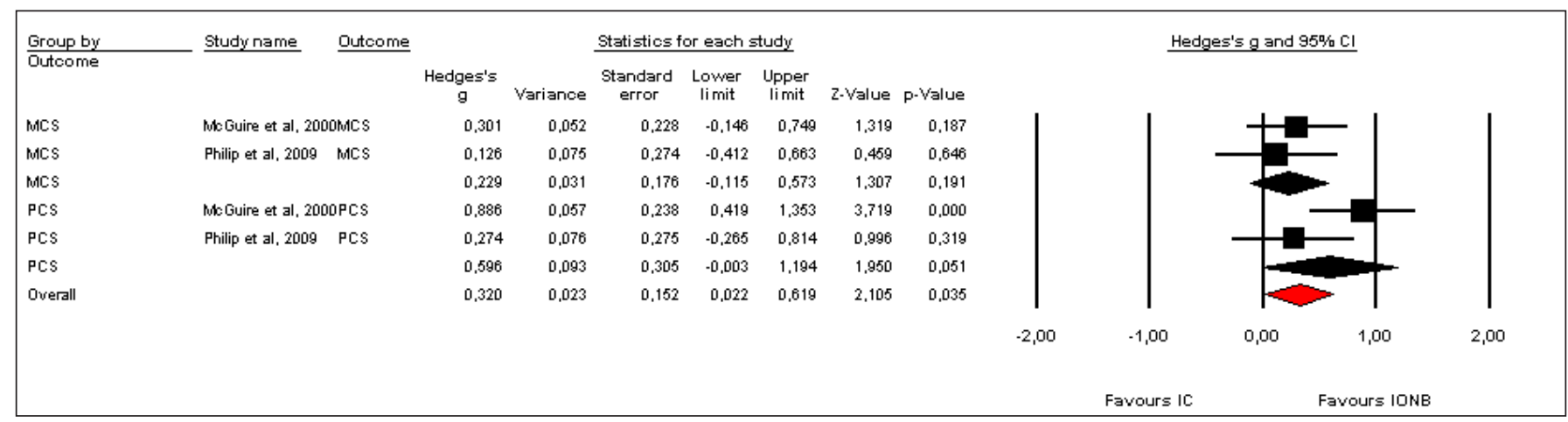

Fig. 5. Forest plot of pooled effect sizes of outcomes from SF-36, analyzing physical and mental components scores. A random-effects model was used for meta-analysis because $\mathrm{I}^{2}=45.705 \%$. Standard mean difference estimates for individual papers, calculated using Hedges' $\mathrm{g}$, are shown in boxes and the summary of effect as a diamond; 95\% CI indicates $95 \%$ confidence interval.

Table 3. Moderator analyses of combined HR-QoL outcomes from all selected articles.

\begin{tabular}{|c|c|c|c|c|}
\hline Moderator & Patients No. & Effects model & $\begin{array}{l}\text { Pooled effect size of } \\
\text { Hedges'g }(95 \% \text { CI; } p)\end{array}$ & I2 for heterogeneity ( $\%)$ \\
\hline \multicolumn{5}{|l|}{ IC mean/median age } \\
\hline$<65$ years & 0 & & & \\
\hline \multicolumn{5}{|l|}{ IONB mean/median age } \\
\hline$<65$ years & 6 & Fixed & $0.309(0.131 / 0.488 ; p=0.001)$ & 14.176 \\
\hline$\geq 65$ years & 4 & Random & $0.153(-0.237 / 0.542 ; \mathrm{p}=0.443)$ & 38.627 \\
\hline \multicolumn{5}{|c|}{ Population mean/median follow-up } \\
\hline$\geq 36$ months & 6 & Fixed & $0.307(0.099 / 0.515 ; \mathrm{p}=0.004)$ & 28.561 \\
\hline \multicolumn{5}{|l|}{ Type of study } \\
\hline Prospective & 0 & & & \\
\hline Retrospective & 10 & Fixed & $0.278(0.125 / 0.431 ; p=0.000)$ & 19.382 \\
\hline \multicolumn{5}{|l|}{ Setting } \\
\hline Multi center & 2 & Fixed & $0.154(-0.233 / 0.541 ; p=0.435)$ & 0 \\
\hline Single center & 8 & Random & $0.291(0.087 / 0.496 ; p=0.005)$ & 31.838 \\
\hline SF36* & 4 & Random & $0.316(0.176 / 0.651 ; p=0.000)$ & 48.352 \\
\hline \multicolumn{5}{|l|}{ Urologic journal } \\
\hline No & 5 & Random & $0.105(-0.225 / 0.436 ; p=0.533)$ & 43.041 \\
\hline Yes & 5 & Fixed & $0.377(0.179 / 0.574 ; p=0.000)$ & 0 \\
\hline \multicolumn{5}{|l|}{ Gender } \\
\hline$>65 \%$ males & 9 & Fixed & $0.291(0.135 / 0.447 ; \mathrm{p}=0.000)$ & 22.856 \\
\hline All females & 1 & Fixed & $-0.085(-0.897 / 0.728 ; \mathrm{p}=0.838)$ & 0 \\
\hline \multicolumn{5}{|l|}{ Publication year } \\
\hline$<2005$ & 4 & Fixed & $0.480(0.265 / 0.696 ; p=0.000)$ & 0 \\
\hline$\geq 2005$ & 6 & Fixed & $0.072(-0.145 / 0.290 ; p=0.516)$ & 0 \\
\hline
\end{tabular}

*Excluding PCS and MCS components scores from Philip et al (20) and McGuire et al (14), the analysis becomes in favour of IONB group [Hedges' $g$ $=0.241(0.070 / 0.411 ; \mathrm{p}=0.006)$.

Follow-up length represents a key-factor in the evaluation of the impact of UD in patients' HR-QoL, because a long coexistence with a UD may be able to change the attitude of patients towards it, becoming part of themselves, establishing a longer practice for the management of it, and affecting the degree of adaptation to their new 


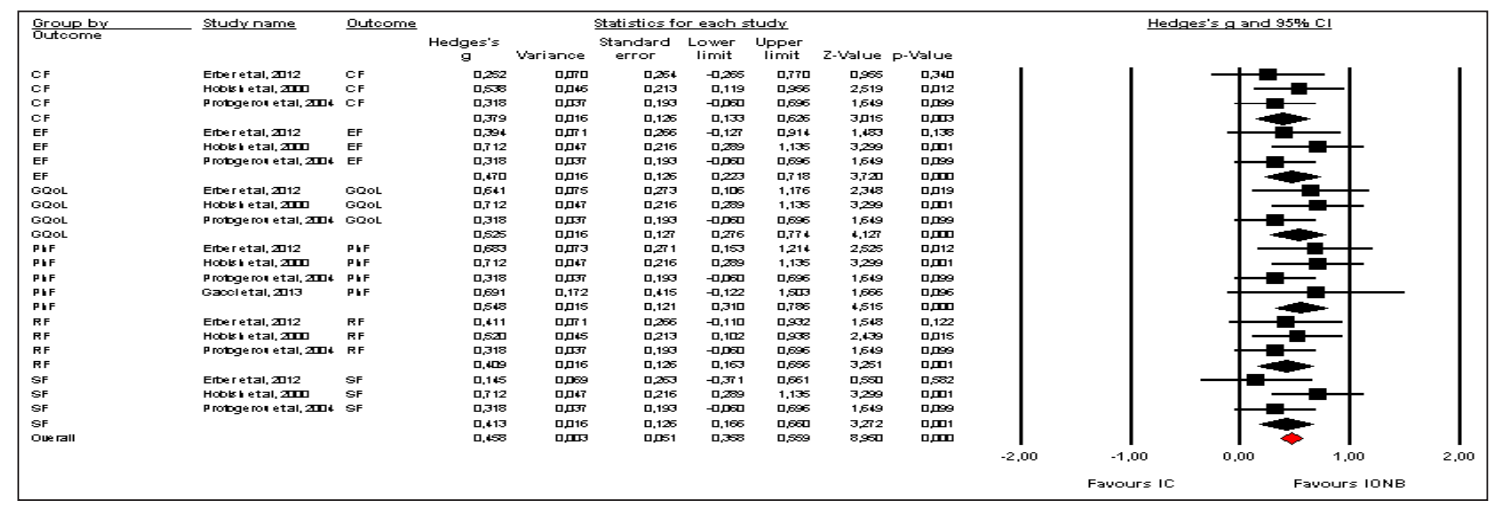

Fig. 6. Forest plot of pooled effect sizes of functional scales from EORTC-QLQ-C30. A fixed-effects model was used for meta-analysis because $\mathrm{I}^{2}=0 \%$. Standard mean difference estimates for individual papers, calculated using Hedges' g, are shown in boxes and the summary of effect as a diamond; 95\% CI indicates 95\% confidence interval.

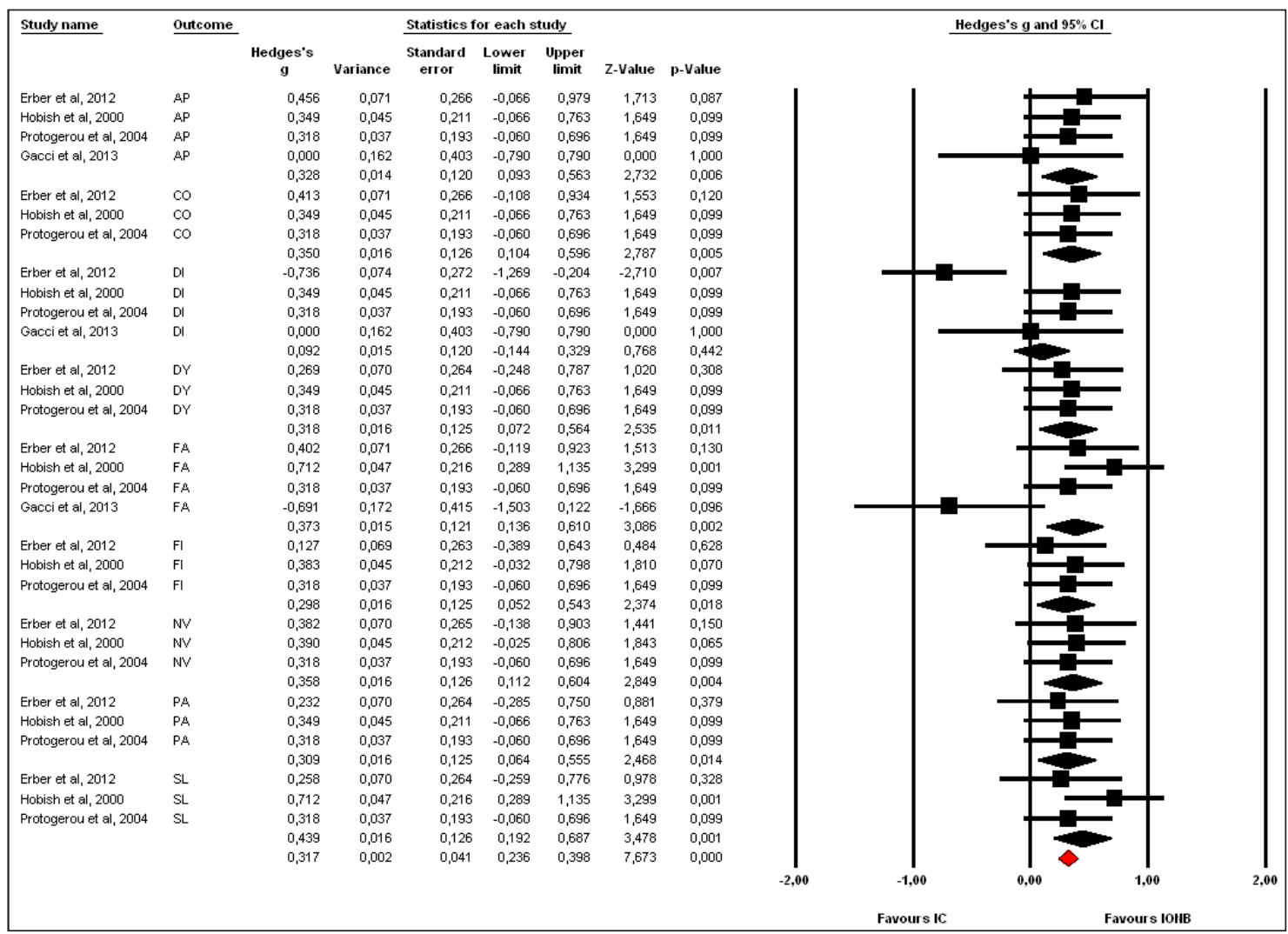

Fig. 7. Forest plot of pooled effect sizes of symptoms scales from EORTC-QLQ-C30. A fixed-effects model was used for meta-analysis because $\mathrm{I}^{2}=4.831 \%$. Standard mean difference estimates for individual papers, calculated using Hedges' $\mathrm{g}$, are shown in boxes and the summary of effect as a diamond; 95\% CI indicates $95 \%$ confidence interval. 


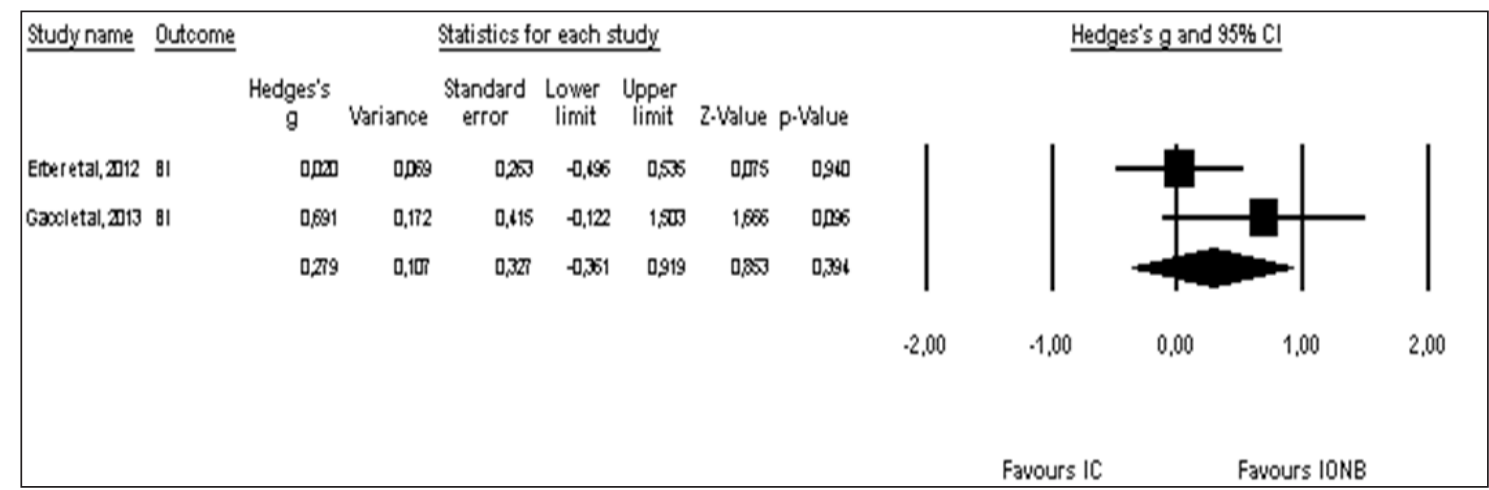

Fig. 8. Forest plot of pooled effect sizes of BI aspect from EORTC-QLQ-BLM30. A random-effects model was used for meta-analysis because $\mathrm{I}^{2}=46.426 \%$. Standard mean difference estimates for individual papers, calculated using Hedges' g, are shown in boxes and the summary of effect as a diamond; 95\% CI indicates $95 \%$ confidence interval.

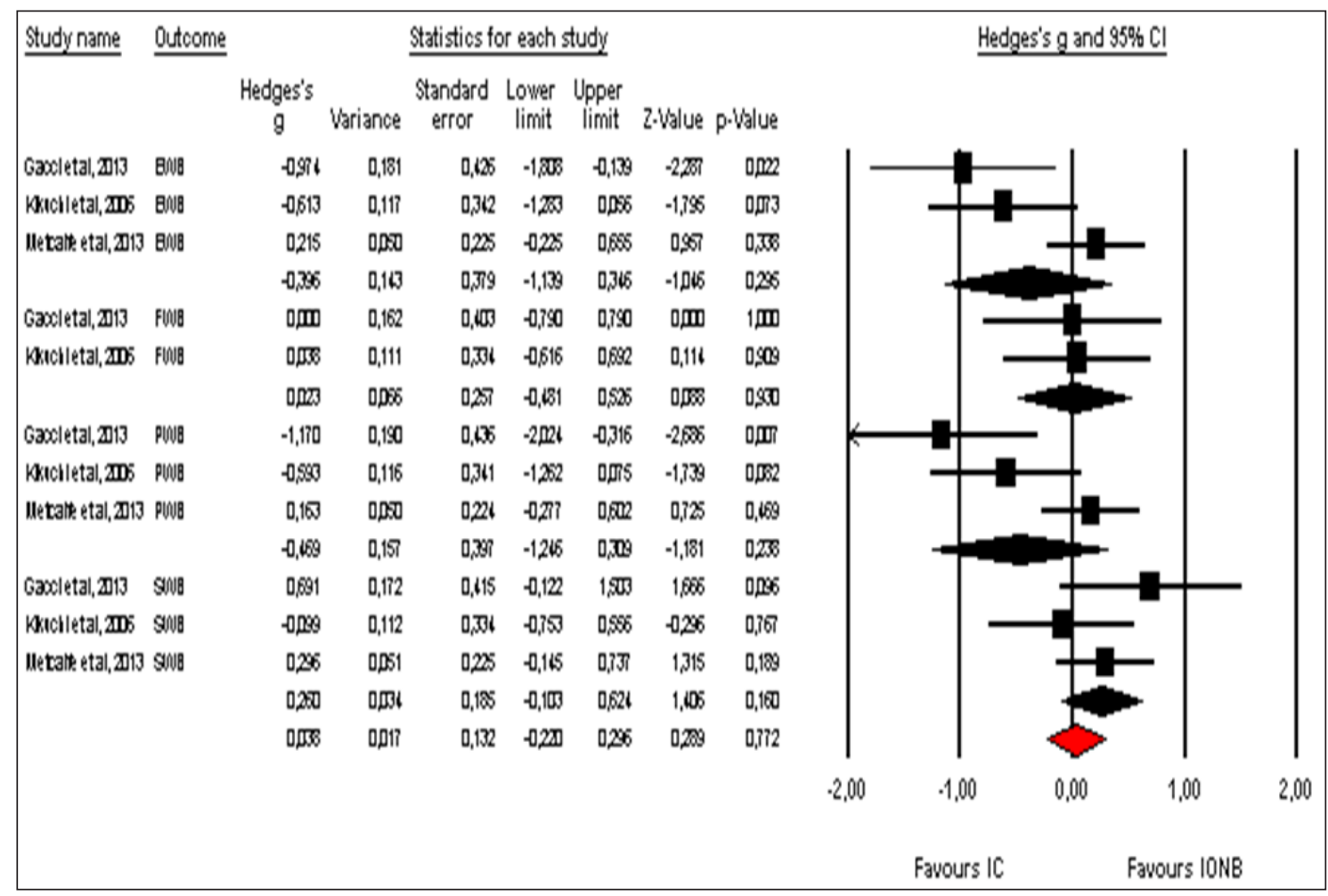

Fig. 9. Forest plot of pooled effect sizes of the FACT-G domains. A random-effects model was used for meta-analysis because $\mathrm{I}^{2}=59.211 \%$. Standard mean difference estimates for individual papers, calculated using Hedges' $\mathrm{g}$, are shown in boxes and the summary of effect as a diamond; 95\% CI indicates $95 \%$ confidence interval.

life with the UD itself [30]. Using a qualitative approach to assess HR-QoL aspects in patients with IC, during the 1 st year after surgery, the experience perceived by patients is better in terms of QoL compared to that perceived after 3-year follow-up because patients are still optimistic about the success of treatment and recovery 
[30]. To obtain a positive prolife a more than 5-year follow-up is needed [30], due to the phenomenon of adaptation. Thus, 36 month follow-up is not a suitable cut-off to analyse follow-up as a significant moderator.

The pooled effect sizes from the studies using of the EORTC QLQ-C30, revealed a superior HR-QoL in bladder cancer patients who received IONB compared to patients who received an IC. Reasons for these discrepant findings are unknown, but may be related to differences in patient characteristics, study design, and/or outcome assessment [23].

Singh et al. [31] documented that, using the EORTC QLQ-C30 in a prospective cohort of patients, at 6,12 and 18 months post operatively, physical functioning ( $\mathrm{p}<$ $0.001, \mathrm{p}<0.001$, and $\mathrm{p}=0.001$, respectively), role functioning $(\mathrm{p}=0.001, \mathrm{p}=0.01$, and $\mathrm{p}=0.003$, respectively), social functioning ( $\mathrm{p}=0.01$ for each follow-up step), and GQoL ( $p<0.001, p<0.001$ and $p=0.002$, respectively) were better in not specify ONB patients. Moreover the financial burden related to bladder cancer treatment was significantly lower in ONB patients in all of analysed follow-up steps $(p=0.05, p=0.05$ and $p=0.005$, respectively). In our study all functional and symptoms score but one (diarrhea) were significantly associated with a better HR-QoL in patients with IONB when compared to IC. In contrast, analyzing papers using the FACT, we did not observe significant differences between the 2 UD subgroups. However it was not possible to perform a comparison between IC and IONB using the FACT items more specific for cystectomy and different UD, due to the lack of published data suitable for meta-analytical evaluation.

Clinicians have presumed that IC patients had a poor body image compared to ONB patients. Actually Kikuchi et al. [18], comparing IC vs. IONB, observed a significant worse HR-QoL score regarding body image in IC subgroup $(\mathrm{p}=0.036)$. In contrast, Somani et al. [32], carrying out a prospective cohort study in order to compare HR-QoL in 29 IC patients vs. 3 ONB ones, at a 12 month follow-up, established that body image did not appear to be an important consideration when patients (already counselled and consented for) are faced with making a decision to have an IC. However they have e very few follow-up and study population to arrive at a definitive conclusion. In our analysis body image did not significantly impact on HR-QoL within the comparison between the analysed UD subgroups.

Our study has important limitations: first, the type of the analyzed studies, not randomized, and retrospective. Second, we did not include studies published in lan-

Health Related Quality of Life of Ileal Conduit vs. Ileal Orthotopic Neobladder guages other than English, unpublished studies, dissertations, or abstracts from conference proceedings. On the other hand, including only published materials ensures that higher quality, peer-reviewed studies were included in the meta-analysis; conversely, excluding unpublished studies is likely to introduce an upward bias into the size of the effects found, which means that calculated effect sizes are likely to be larger. Addressing this limitation, we did not observe significant publication bias. Third, given the number of moderators and the multiple outcomes we tested, we had a high chance of incidental findings of statistically significant moderators. To account for this bias, we just interpreted moderators with $\geq$ 5 studies involved, which were significant at a 0.05 level for an overall outcome and not those that were significant for a subgroup within particular outcome. Forth, each of the moderators was examined in separate analyses. We did not assess multiple moderators in one meta-regression model due to the small number of studies for each outcome. All the above mentioned limitations have to be considered when interpreting the findings.

\section{Conclusion}

This meta-analysis of not-randomized, retrospective comparative studies on the impact of IC vs. IONB on HR-QoL showed a significant advantage of the IONB subgroups. Because of the presence of heterogeneity in several aspects of the analyzed papers, in order to corroborate the arguments in favour of the IONB as the urinary diversion of choice, randomized controlled trials comparing different types of UD using validated, disease-specific HR-QoL tools are needed, whenever technically feasible and oncologically justified. Alternatively well designed prospective cohort studies with UD subgroups suitably matched for age, gender, type of validated HR-QoL questionnaire, and with a long follow-up are needed. 


\section{References}

1 Stenzl A, Cowan NC, De Santis M, Kuczyk MA, Merseburger AS, Ribal MJ, Sherif A, Witjes JA: Treatment of muscle-invasive and metastatic bladder cancer: update of the EAU guidelines. Eur Urol 2011;59:1009-1018.

2 Rouprêt M, Zigeuner R, Palou J, Boehle A, Kaasinen E, Sylvester R, Babjuk M, Oosterlinck W: European guidelines for the diagnosis and management of upper urinary tract urothelial cell carcinomas: 2011 update. Eur Urol 2011;59:584-594.

3 Hautmann RE: Urinary diversion: ileal conduit to neobladder. J Urol 2003;169:834-842.

4 Lee CT: Quality of life following incontinent cutaneous and orthotopic urinary diversions. Curr Treat Options Oncol 2009;10:275-286.

5 Arata R, Saika T, Tsushima T, Abarzua F, Nasu Y, Kumon H: Orthotopic ileal neoblader versus sigmoidal neobladder: a "quality of life" (QOL) survey. Acta Med Okayama 2007;61:229-234.

6 Miyake H, Furukawa J, Muramaki M, Inoue T, Fujisawa M: Health related quality of life after radical cystectomy: comparative study between orthotopic sigmoid versus ileal neobladders. Eur J Surg Oncol 2012;38: 1089-1094.

7 Miyake H, Furukawa J, Sakai I, Muramaki M, Yamashita M, Inoue T, Fujisawa M: Orthotopic sigmoid vs ileal neobladders in Japanese patients: a Comparative assessment of complications, functional outcomes, and quality of life. Urol Oncol 2013;31:11551160.

8 Ali AS, Hayes MC, Birch B, Dudderidge T, Somani BK: Health related quality of life (HRQoL) after cystectomy: comparison between orthotopic neobladder and ileal conduit diversion. Eur J Surg Oncol 2015;41: 295-299.

9 Borenstein M, Hedges LV, Higgins JPT, Rothstein HR: Introduction to Meta-Analysis. Chichester, UK, John Wiley \& Sons Ltd, 2009.

10 Higgins JP, Thompson SG: Quantifying heterogeneity in a meta-analysis. Stat Med 2002;21:1539-1558.

11 Higgins JP, Thompson SG, Deeks J, Altman DG: Measuring inconsistency in meta-analyses. BMJ 2003;327:557-560.

12 Moher D, Liberati A, Tetzlaff J, Altman DG: Preferred reporting items for systematic reviews and meta-analyses: the PRISMA statement. PLoS Med 2009;6:e1000097.

13 Cohen J: Statistical Power Analysis for the Behavioral Sciences, ed 2. Hillsdale, NJ: Lawrence Erlbaum, 1988.
14 McGuire MS, Grimaldi G, Grotas J, Russo $P$ : The type of urinary diversion after radical cystectomy significantly impacts on the patient's quality of life. Ann Surg Oncol 2000; $7: 4-8$.

15 Hobisch A, Tosun K, Kinzl J, Kemmler G, Bartsch G, Höltl L, Stenzl A: Quality of life after cystectomy and orthotopic neobladder versus ileal conduit urinary diversion. World J Urol 2000;18:338-344.

16 Dutta SC, Chang SS, Coffey CS, Smith JA Jr, Jack G, Cookson MS: Health related quality of life assessment after radical cystectomy: comparison of ileal conduit with continent orthotopic neobladder. J Urol 2002;168:164167.

17 Protogerou V, Moschou M, Antoniou N, Varkarakis J, Bamias A, Deliveliotis C: Modifies S-pouch neobladder vs ileal conduit and a matched control population: a quality of life survey. BJU Int 2004;94:350-354.

18 Kikuchi E, Horiguchi Y, Nakashima J, Ohigashi T, Oya M, Nakagawa K, Miyajima A, Murai M: Assessment of long-term quality of life using the FACT-BL questionnaire in patients with an ileal conduit, continent reservoir, or orthotopic neobladder. Jpn J Clin Oncol 2006;36:712-716.

19 Autorino R, Quarto G, Di Lorenzo G, De Sio $\mathrm{M}$, Perdonà S, Giannarini G, Giugliano F, Damiano R: Health related quality of life after radical cystectomy: comparison of ileal conduit to continent orthotopic neobladder. Eur J Surg Oncol 2009;35:858-864.

20 Philip J, Manikandan R, Venugopal S, Desouza J, Javlè PM: Orthotopic neobladder versus ileal conduit urinary diversion after cystectomy - a quality-of-life based comparison. Ann R Coll Surg Engl 2009;91:565-569.

21 Erber B, Schrader M, Miller K, Schostak M, Baumunk D, Lingnau A, Schrader AJ, Jentzmik F: Morbidity and quality of life in baldder cancer patients following cystectomy and urinary diversion: a single-institution comparison of ileal conduit versus orthotopic neobladder. ISRN Urology 2012; 2012:342796

22 Gacci M, Saleh O, Cai T, Gore JL, D'Elia C, Minervini A, Masieri L, Giannessi C, Lanciotti M, Varca V, Simonato A, Serni S, Carmignani G, Carini M: Quality of life in women undergoing urinary diversion for bladder cancer: results of a multi center study among long-term disease-free survivors. Health Qual Life Outcomes 2013;11:43.

23 Metcalfe M, Estey E, Jacobsen NE, Voaklander D, Fairey AS: Association between urinary diversion and quality of life after radical cystectomy. Can J Urol 2013;20:66266631.
24 Ware JE Jr, Sherbourne CD: The MOS 36item short forma health survey (SF-36): I. Conceptual framework and item selection. Med Care 1992;30:473-483.

25 Stansfeld SA, Roberts R, Foot SP: Assessing the validity of the SF-36 general health survey. Qual Life Res 1997;6:217-224.

26 Cookson MS, Dutta SC, Chang SS, Clark T, Smith JA Jr, Wells H: Health related quality of life in patients treated with radical cystectomy and urinary diversion for urotelial carcinoma of the bladder: development of a new disease specific questionnaire. J Urol 2003;170:1926-1930.

27 Saika T, Arata R, Tsushima T, Nasu Y, Suyama B, Takeda K, Ebara S, Manabe D, Kobayashi, Tanimoto R, Kumon H; Okayama Urological Research Group: Health-related quality of life after radical cystectomy for bladder cancer in elderly patients with an ileal conduit, ureterocutaneostomy, or orthotopic urinary reservoir: a comparative questionnaire survey. Acta Med Okayama 2007; 61:199-203.

28 Sogni F, Brausi M, Frea B, Martinengo C, Faggiano F, Tizzano A, Gontero P: Morbidity and quality of life in elderly patients receiving ileal conduit or orthotopic neobladder after radical cystectomy for invasive bladder cancer. Urology 2008;71:919-923.

29 Rouanne M, Legrand G, Neuzillet Y, Ghoneim T, Cour F, Letang N, Yonneau L, Hervé JM, Botto H, Lebret T: Long-term women-reported quality of life after radical cystectomy and orthotopic ileal neobladder reconstruction. Ann Surg Oncol 2014;21:1398-1404.

30 Cerruto MA, D'Elia C, Cacciamani G, De Marchi D, Siracusano S, Iafrate M, Niero M, Lonardi C, Bassi P, Belgrano E, Imbimbo C, Racioppi M, Talamini R, Ciciliato S, Toffoli L, Rizzo M, Visalli F, Verze P, Artibani W: Behavioural profile and human adaptation of survivors after radical cystectomy and ileal conduit. Health Qual Life Outcomes 2014;12:46.

31 Singh V, Yadav R, Sinha RJ, Gupta DK: Prospective comparison of quality-of-life outcomes between ileal conduit urinary diversion and orthotopic neobladder reconstruction after radical cystectomy: a statistical model. BJU Int 2014;113:726-732.

32 Somani BK, Gimlin D, Fayers P, N'Dow J: Quality of life and body image for bladder cancer patients undergoing radical cystectomy and urinary diversion - a prospective cohort study with a systematic review of the literature. Urology 2009;74:1138-1144. 